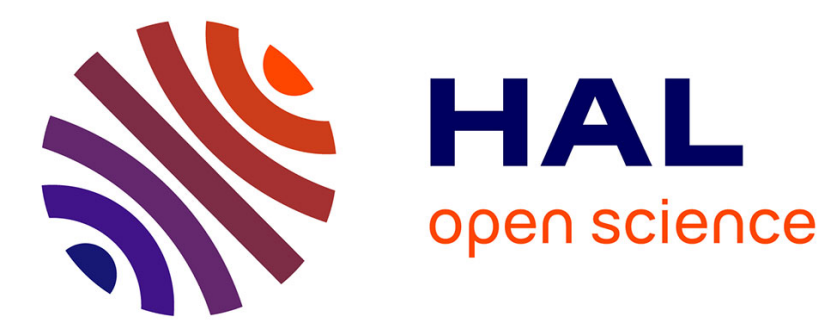

\title{
Critical current density determination of superconducting material
}

Bruno Douine, Kévin Berger, Jean Lévêque

\section{To cite this version:}

Bruno Douine, Kévin Berger, Jean Lévêque. Critical current density determination of superconducting material. CISTEM 2014 - Conférence Internationale en Sciences et Technologies Electriques au Maghreb, Nov 2014, Tunis, Tunisie. pp.1-5, 10.1109/CISTEM.2014.7076967 . hal-01280968

\section{HAL Id: hal-01280968 https://hal.science/hal-01280968}

Submitted on 2 Mar 2016

HAL is a multi-disciplinary open access archive for the deposit and dissemination of scientific research documents, whether they are published or not. The documents may come from teaching and research institutions in France or abroad, or from public or private research centers.
L'archive ouverte pluridisciplinaire HAL, est destinée au dépôt et à la diffusion de documents scientifiques de niveau recherche, publiés ou non, émanant des établissements d'enseignement et de recherche français ou étrangers, des laboratoires publics ou privés. 


\title{
Critical current density determination of superconducting material
}

\author{
Bruno Douine, Kévin Berger, Jean Lévêque \\ GREEN Université de Lorraine \\ Vandoeuvre-lès-Nancy \\ bruno.douine@univ-lorraine.fr
}

\begin{abstract}
Knowledge of the law critical current density versus magnetic field, $J_{C}(B)$, of a superconducting material is very important to users. In this article are presented two methods of characterization to obtain experimentally the law $\mathbf{J}_{C}(B)$. These two methods are commonly used for applications in electrical engineering. The electrical method is based on measuring the voltage and current of a superconducting sample. It has the advantage of simplicity in implementation, but the main drawback as the presence of the self magnetic field. This prevents the determination of the parameters of $J_{C}(B)$ for weak magnetic fields. The magnetic method using the cycle of magnetization has the advantage of making measurements without contact with the sample but has two drawbacks: it is based on a theoretical model in which $J_{C}$ is constant and the assumption of infinite length sample.
\end{abstract}

Keywords-characterization, critical current density, superconducting material.

\section{INTRODUCTION}

Les matériaux supraconducteurs ont la propriété de transporter du courant avec des densités de courant beaucoup plus fortes que les conducteurs classiques. De plus, leurs pertes en courant continu sont quasi nulles et en courant alternatif plus faibles que les conducteurs classiques. Ces matériaux sont caractérisés par trois paramètres qui sont la température critique $T_{C}$, le champ magnétique critique $H_{C}$ et la densité de courant critique $J_{C}[1 ; 2]$. Dans les applications utilisant ces matériaux supraconducteurs tels que, la création de fort champ magnétique, la lévitation magnétique, le transport de courant, les moteurs et transformateurs, la connaissance de $J_{C}$ du matériau est primordiale. Ainsi dans les bobines de champ $J_{C}$ est liée au champ magnétique maximum créé, dans les systèmes de lévitation $J_{C}$ fixe le pouvoir de lévitation, dans les câbles de courant de transport $J_{C}$ détermine les pertes qui limitent la valeur du courant, enfin dans les moteurs le couple est directement lié à $J_{C}$. La densité de courant critique est dépendant des deux autres paramètres c'est à dire le champ magnétique $B$ et la température $T$, on cherche donc à déterminer la loi $J_{C}(B, T)$. Dans cet article on se limitera à la détermination de la loi $J_{C}(B)$ à $T$ constant, ce qui reste un problème ouvert notamment pour les supraconducteurs à haute température critique (HTC).

Dans les supraconducteurs, la relation entre $\mathrm{J}$ et $\mathrm{E}$ peut être mise sous la forme :

$$
\vec{E}=E_{C} \cdot\left(\frac{J}{J_{C}(B, T)}\right)^{n(B, T)} \cdot \vec{J}
$$

Pour les supraconducteurs à basse température critique, l'exposant $n$ est grand et vaut plusieurs dizaines. Mais pour les supraconducteurs à haute température critique $n$ varie entre 7 et 30. La valeur de $\mathrm{n}$ est déterminée à partir de courbes expérimentales. Si $n$ tend vers l'infini on retrouve le modèle de l'état critique créé par Bean [3]. Celui-ci impose que la densité locale de courant dans un matériau supraconducteur est soit nulle, soit égale à sa valeur critique $J_{C}$

$$
J= \pm J_{C} \text { oи } J=0
$$

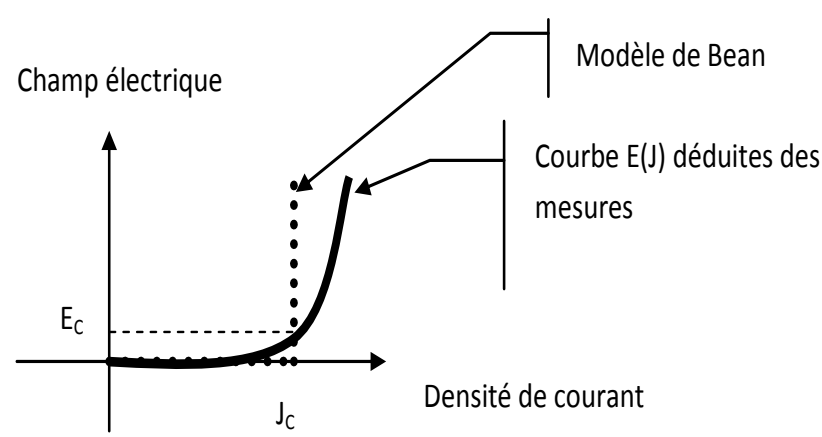

Fig. 1. Courbe E(J) d'un supraconducteur

Les relations $J_{C}(B)$ utilisées les plus couramment dans la littérature sont (tableau 1) les modèles de Bean [3] , de Kim [4] et linéaire $[5 ; 6]$. Le modèle de Bean est très utilisé dans les calculs théoriques car il permet des calculs analytiques simples de répartition de courant dans un matériau supraconducteur comme par exemple dans une plaque ou un cylindre. Le modèle linéaire est valable surtout pour les supraconducteurs à basse température critique (BTC) et permet des calculs analytiques de pertes [7]. Le modèle de Kim est très utilisé car il est le plus proche des mesures expérimentales. 
TABLEAU 1 : Les relations $J_{C}(B)$ utilisées les plus couramment

\begin{tabular}{|c|c|}
\hline $\mathrm{J}_{\mathrm{CB}}=\mathrm{J}_{\mathrm{C} 0}$ independant of B & $\begin{array}{c}\text { Bean } \\
{[3]}\end{array}$ \\
\hline $\mathrm{J}_{\mathrm{CK}}(\mathrm{B})=\frac{\mathrm{J}_{\mathrm{C} 0}}{\left(1+\frac{|\mathrm{B}|}{\mathrm{B}_{\mathrm{K} 0}}\right)}$ & $\begin{array}{c}\text { Kim } \\
{[4]}\end{array}$ \\
\hline $\mathrm{J}_{\mathrm{CL}}(\mathrm{B})=\frac{\mathrm{J}_{\mathrm{C} 0}\left(\mathrm{~B}_{\mathrm{j} 0}-|\mathrm{B}|\right)}{\mathrm{B}_{\mathrm{j} 0}}$ & Linéaire \\
& {$[5]$} \\
\hline
\end{tabular}

$\mathrm{J}_{\mathrm{C} 0}, \mathrm{~B}_{\mathrm{K} 0}$ and $\mathrm{B}_{\mathrm{J} 0}$ sont des constantes positives.

La loi $J_{C}(B)$ d'un matériau supraconducteur peut-être déterminer de plusieurs façons [8; 9]. Dans cet article seront présentées une méthode électrique, où l'échantillon est en contact avec le système de mesure on parle alors de méthode directe, et une méthode magnétique, où l'échantillon n'est pas en contact avec le système de mesure on parle alors de méthode indirecte.

\section{METHODE ELECTRIQUE DE DETERMINATION EXPERIMENTALE DE LA DENSITE DE COURANT CRITIQUE}

Les mesures électriques de densité de courant critique des matériaux supraconducteurs sont habituellement accomplies par la méthode de mesure 4-points [6;9]. L'échantillon est alimenté par un courant continu I et une différence tension $U$ aux bornes de l'échantillon est relevée par deux fils indépendants des amenées de courant. De manière simple voire simpliste on tire $E$ de $U$ par $U=E . L$ avec $L$ la distance entre les prises de potentiel et $J$ de $I$ par $J=I / S$ avec $S$ la section de l'échantillon. Ces relations ne sont valables théoriquement que si la densité de courant a entièrement pénétré l'échantillon c'est à dire dans le cas où le courant est suffisamment grand mais on utilise en général ces relations aussi en faible courant. De plus, pour les courants forts, des effets thermiques dus aux pertes dans le matériau supraconducteur et dans les résistances de contact supra-cuivre empêchent de réaliser des mesures parfaitement isothermes. Il faut donc rester prudent sur les courbes $E(J)$ déduites de ces mesures. De $E(J)$ on tire la valeur de $J_{C}$ en appliquant un critère de champ électrique de $1 \mu \mathrm{V} / \mathrm{cm}$. Pour obtenir la courbe $J_{C}(B)$ expérimentale, l'échantillon alimenté avec un courant I est soumis à un champ magnétique extérieur $B_{\text {ext }}$ parallèle à son axe (Fig. 2). La tension $U$ est mesurée en fonction de I pour différents champs magnétiques extérieurs $B_{e x t}$ (Fig. 6). Pour différencier la relation $J_{C}(B)$ locale présente dans le matériau de celle déduite des mesures cette dernière est notée $J_{m c}\left(B_{e x t}\right)$ par la suite".

Des mesures ont été faites (figure 3) sur un tube HTC de BiSCCO dont les dimensions sont les suivantes : rayon intérieur $R_{i}=3,8 \mathrm{~mm}$, rayon extérieur $R_{e}=5 \mathrm{~mm}$, section $S=$ $33 \mathrm{~mm}^{2}$ et distance entre les prises de potentiel $\mathrm{h}=11,7 \mathrm{~cm}$. Hors champ magnétique extérieur, le courant critique mesuré est égale à $I_{\mathrm{mc} 0}=96 \mathrm{~A}$, avec un critère de $1 \mu \mathrm{V} / \mathrm{cm}$.

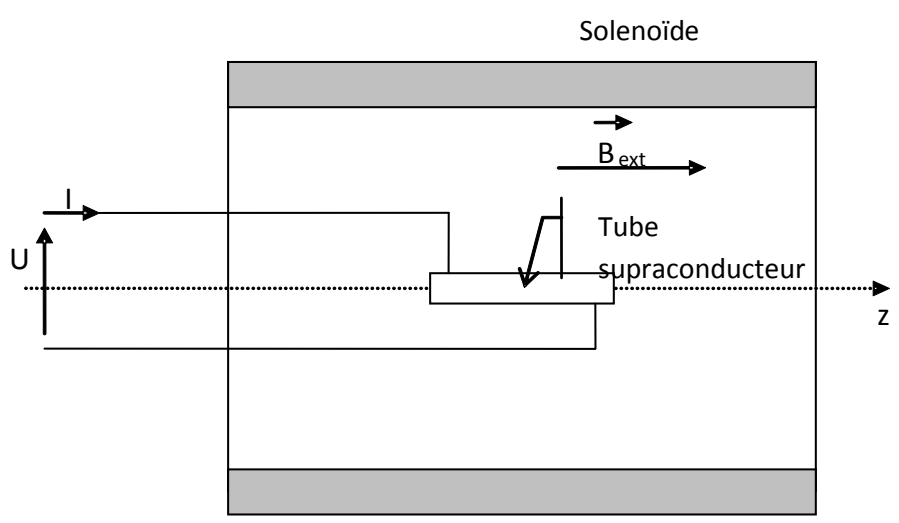

Fig. 2. Banc de mesure de $J_{C}(B)$ par la méthode électrique

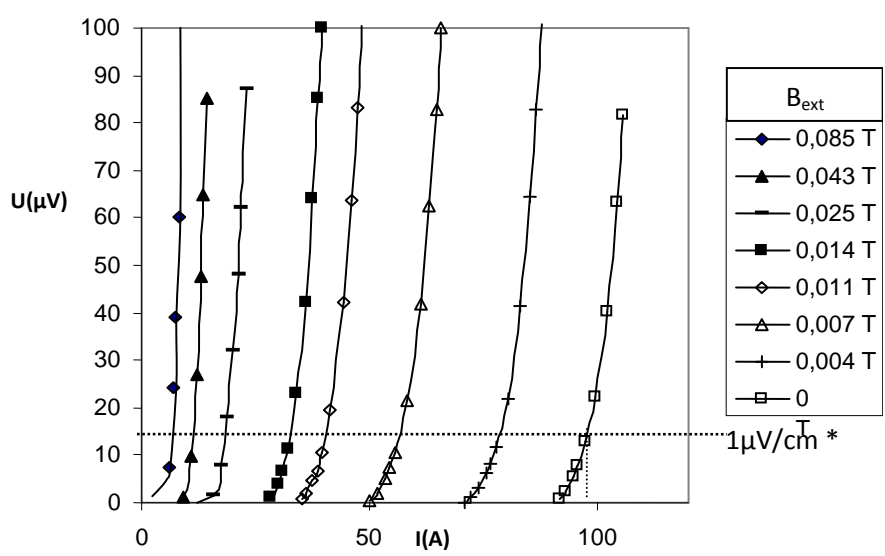

Fig. 3. Courbes U(I) pour différents $B_{\text {ext }}$
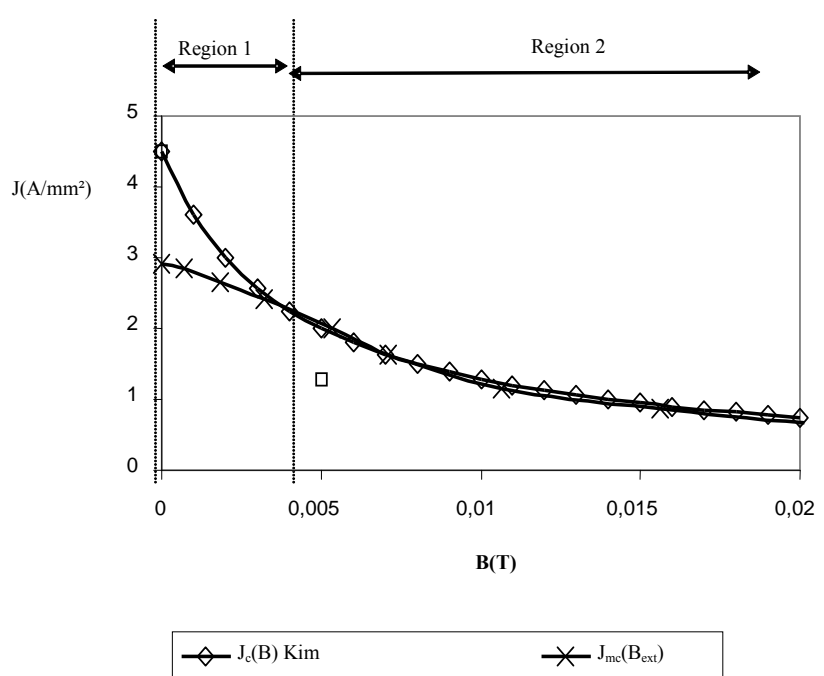

Fig. 4. Courbe $J_{C}(B)$ expérimentale et courbe théorique extrapolée à l'aide du modèle de $\operatorname{Kim} U(I)$ pour différents $B_{\text {ext }}$

$J_{m c}\left(B_{e x t}\right)$ et $J_{C}(B)$ sont différents car :

- $J_{C}(B)$ est une grandeur microscopique et $J_{m c}\left(B_{e x t}\right)$ est une grandeur macroscopique 
- $B$ n'est pas égal à $B_{\text {ext }}$ à cause du champ magnétique propre $B_{S F}, \vec{B}=\vec{B}_{S F}+\vec{B}_{\text {ext }}, B=\sqrt{B_{S F}^{2}+B_{e x t}^{2}}$

Quand la valeur de $B_{e x t}$ est suffisamment grande, $B_{S F}$ devient négligeable et les valeurs de $J_{m c}\left(B_{e x t}\right)$ sont très proches de $J_{C}(B)$ (Fig. 4, région 2). Au contraire quand $B_{\text {ext }}$ est proche de $B_{S F}, J_{m c}\left(B_{e x t}\right)$ peut-être très différent de $J_{C}(B)$ (Fig. 4, région $1)$ Sur la Fig. 4 sont représentés $J_{m c}\left(B_{e x t}\right)$ et la loi $J_{C}(B)$ théorique tiré du modèle de Kim et qui correspond le mieux aux mesures dans la région 2:

$$
J_{c}(B)=\frac{4.6 .10^{6}}{1+\frac{|B|}{0.004}} \quad A / m^{2}
$$

Le modèle de Kim a été choisi car il est le plus approprié pour décrire le comportement de $J_{C}(B)$ dans le cas des composés granulaire de BiSCCO [8].

\section{METHODE MAGNETIQUE DE DETERMINATION EXPERIMENTALE DE LA DENSITE DE COURANT CRITIQUE}

La méthode électrique ou méthode directe nécessite de connecter l'échantillon supraconducteur à une alimentation de courant. Ceci introduit des problèmes thermiques du fait de l'apport d'énergie importante à basse température. Pour éviter ce problème des méthodes sans connections électriques dite méthodes indirectes ou méthodes magnétiques existent. Elles sont de deux types, la méthode DC et la méthode AC. Cette dernière ne sera pas décrite dans cet article mais le lecteur pourra la retrouver dans un certain nombre de références $[8 ; 9]$. Le principe de la méthode DC est le suivant. L'échantillon supraconducteur est soumis à un champ magnétique appliqué $H_{a}$ qui provoque l'apparition de courant induit d'écrantage dans l'échantillon. Ces courants induits créent un moment magnétique $\mathrm{m}$ que l'on assimile à une "aimantation" $M$ avec $M=m / V$ ( $V$ le volume de matériau supraconducteur). Le champ magnétique local $H$ est la somme vectorielle du champ magnétique appliquée et du champ induit $H_{i}$ créé par les supracourants:

$$
\vec{H}=\overrightarrow{H_{a}}+\overrightarrow{H_{i}}
$$

On calcule l'induction magnétique moyenne $\langle B\rangle$ qui est la somme du champ magnétique appliquée est de "l'aimantation" du matériau :

$$
<B>=\mu_{0} H_{a}+\mu_{0} M
$$

Pour obtenir $M$ expérimentalement la méthode est la suivante. L'échantillon supraconducteur est placé au bout d'une tige relié à un moteur permettant un mouvement linéaire et dans une région soumis à un champ appliqué $H_{a}$ quasi uniforme (Fig. 5). La mesure se fait en deux phases. Première phase un champ magnétique est appliqué progressivement.

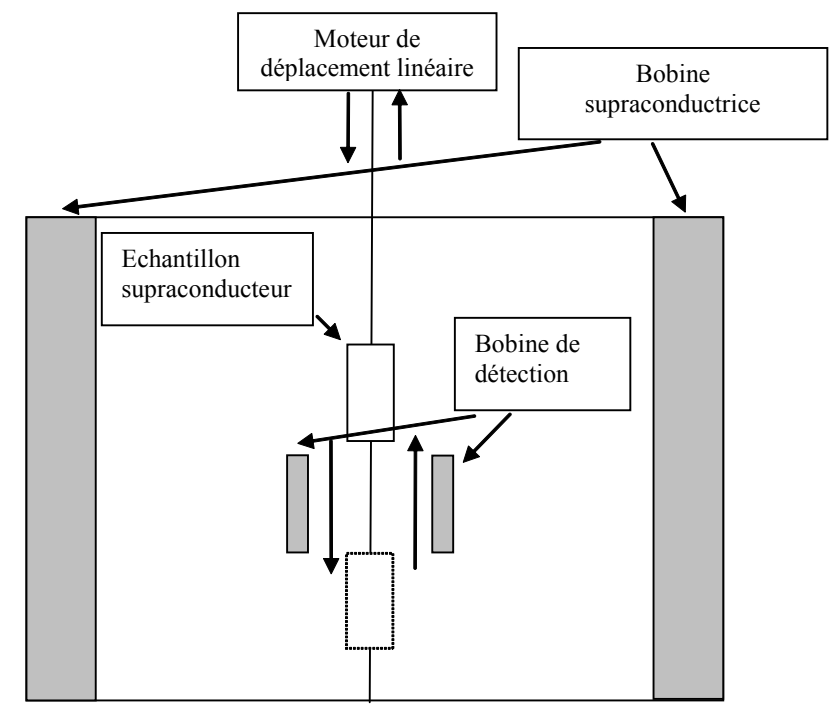

Fig. 5. Banc de mesure de $M\left(H_{a}\right)$

Deuxième phase le champ magnétique est maintenu constant et l'échantillon est déplacé de bas en haut. Une tension induite $e(t)$ due au supracourant induit dans le matériau est enregistrée par le système de mesure. La courbe $M\left(H_{a}\right)$ en est déduite (Fig. 6) à l'aide de la formule suivante :

$$
\mathrm{M}=\frac{<\mathrm{B}>}{\mu_{0}}-\mathrm{H}_{\mathrm{a}}=-\frac{\varphi_{\max }}{\mathrm{S} \cdot \mu_{0}}-\mathrm{H}_{\mathrm{a}}=\frac{\int \mathrm{e}(\mathrm{t}) \cdot \mathrm{dt}}{\mathrm{S} \cdot \mu_{0}}-\mathrm{H}_{\mathrm{a}}(6)
$$

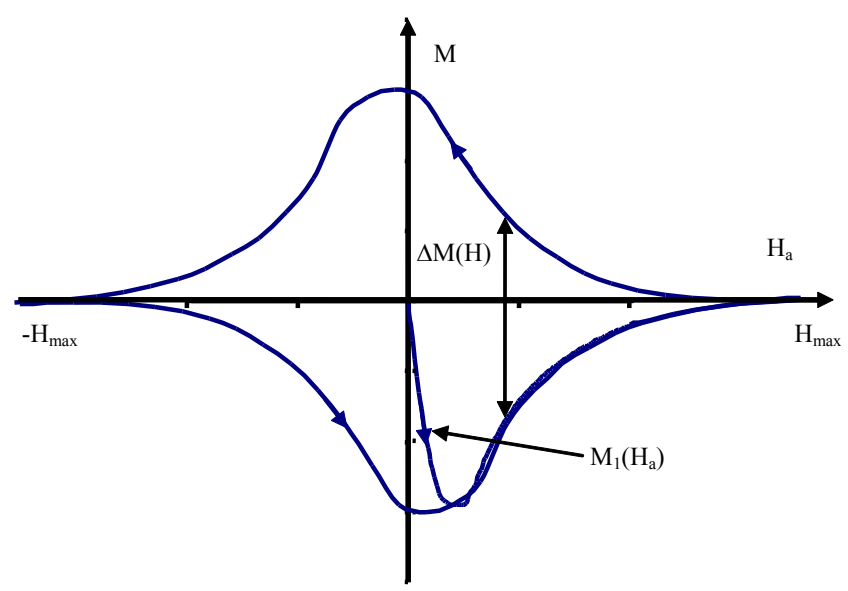

Fig. 6. Courbe expérimentale $M\left(H_{a}\right)$

L'échantillon utilisé ici (Fig.6) est un cylindre de NbTi de $2 \mathrm{~cm}$ de long et de $5 \mathrm{~mm}$ de diamètre à une température de $5^{\circ} \mathrm{K}$.

Cette courbe $M\left(H_{a}\right)$ est constituée de deux parties. Premièrement, une courbe de première aimantation $M_{l}\left(H_{a}\right)$ qui correspond à la première augmentation de $\mathrm{H}_{\mathrm{a}}$. Elle commence alors que le matériau supraconducteur ne contient aucun courant et se termine pour $H_{a}=H_{\max }$. Deuxièmement, un cycle d'hystérésis pour $H_{a}$ allant de $H_{\max } \grave{a}-H_{\max }$ puis de $-H_{\max } \grave{a}$ $H_{\max }$. 
Actuellement le modèle le plus utilisé pour déduire $J_{C}(B)$ de $M\left(H_{a}\right)$ est le modèle de l'état critique. Nous rappelons que nous appelons "modèle de Bean" (tableau 1), pour la loi $J_{C}(B)$, une relation où $J_{C}$ est constante et égale à $J_{C 0}$. Dans le cas d'un échantillon supraconducteur cylindrique soumis à un champ axial le modèle de l'état critique impose une relation simple entre $J_{C}$ et $B$ :

$$
\frac{d B}{d r}=\mu_{0} J_{C}
$$

A l'aide de (6) et de (7) on déduit la courbe théorique $M\left(H_{a}\right)$ (Fig. 17). Elle est constituée d'une courbe de première aimantation $M_{1 B}\left(H_{a}\right)$ et d'un cycle d'hystérésis. La courbe théorique de première aimantation est elle aussi décomposée en deux parties. En dessous de $H_{P B}$ il y a pénétration incomplète du champ dans l'échantillon (indice I), au dessus de $\mathrm{H}_{\mathrm{PB}}$ il y a pénétration complète (indice $\mathrm{C}$ ):

$$
\begin{gathered}
M_{1 B I}\left(H_{a}\right)=-\frac{H_{a}\left(\left(\mu_{0} H_{a}\right)^{2}-\mu_{0}^{2} H_{a} . J_{C 0} R+3\left(\mu_{0} J_{C 0} R\right)^{2}\right)}{3\left(\mu_{0} J_{C 0} R\right)^{2}} \\
M_{1 B C}\left(H_{a}\right)=-\frac{J_{C 0} R}{3}=-\frac{H_{P B}}{3}
\end{gathered}
$$

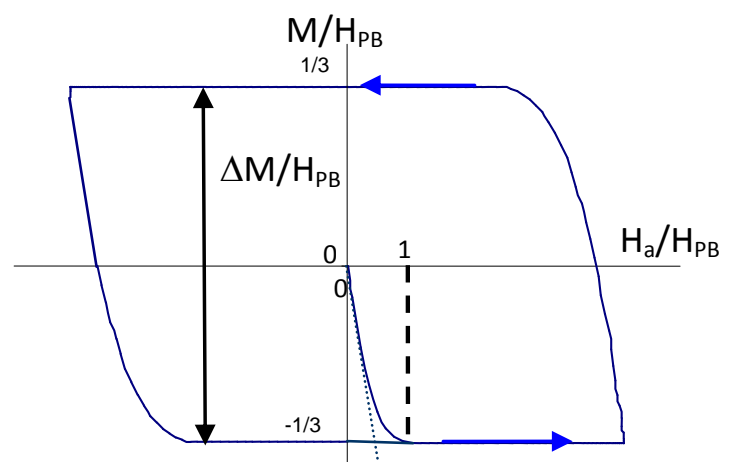

Fig. 7. Courbe théorique $M\left(H_{a}\right)$

Il existe donc une relation très simple entre la largeur du cycle $\Delta M$ et $J_{C}$ :

$$
J_{C}=J_{C 0}=\frac{3 \Delta M}{2 R}
$$

La méthode traditionnelle de détermination de $J_{C}(B)$ [8;9] à l'aide du cycle $M\left(H_{a}\right)$ utilise donc [10], tiré du modèle de Bean où $J_{C}$ est constant, en l'extrapolant aux cas où $J_{C}$ dépend de $B$ :

$$
J_{C}(B)=\frac{3 \Delta M(H)}{2 R}
$$

Sur la Fig. 8 est représentée la courbe $J_{C}(B)$ du matériau dont la courbe $M\left(H_{a}\right)$ est représentée sur la Fig. 6 en utilisant la méthode traditionnelle présentée ici et comparée avec une méthode par identification développée dans [11]. L'échantillon est un cylindre $\mathrm{NbTi}$ de $2 \mathrm{~cm}$ de long et de $5 \mathrm{~mm}$ de diamètre à une température de $5^{\circ} \mathrm{K}$.

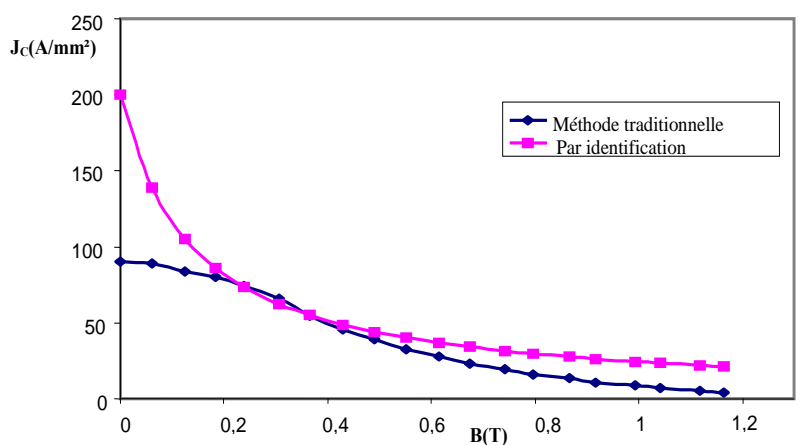

Fig. 8. Courbes $J_{C}(B)$ expérimentales, l'une tirée du modèle de Bean et l'autre à l'aide d'une méthode d'identification

\section{CONCLUSION}

La connaissance la plus précise possible de la loi $J_{C}(B)$ d'un matériau supraconducteur est très importante pour les utilisateurs. Dans cet article sont exposées deux méthodes de caractérisation permettant d'obtenir expérimentalement cette loi $J_{C}(B)$. La méthode électrique a comme avantage sa simplicité de mise en œuvre mais comme inconvénient principale la présence du champ propre qui empêche la détermination des paramètres de $J_{C}(B)$ pour les champs magnétiques faibles. Une première réponse à ce problème a été présentée mais ne permet qu'une compensation partielle du champ propre. La méthode magnétique utilisant le cycle d'aimantation a comme avantage de réaliser des mesures sans contacts avec l'échantillon mais a deux inconvénients; il est basé sur un modèle théorique dans lequel $J_{C}$ est constant et sur l'hypothèse d'échantillons de longueur infinie. Des éléments de réponses à ces problèmes ont été proposés. Il faut mettre en garde le lecteur sur le fait que chaque méthode de caractérisation des supraconducteurs est adaptée à un type d'application. Par exemple pour des câbles de transport de courant la méthode électrique est plus appropriée car il s'agit dans ce cas de connaître le courant que l'on pourra injecter dans ce câble. Deuxième exemple, pour un écran supraconducteur il est indispensable d'estimer le champ magnétique qu'il sera possible d'écranter et la méthode magnétique est alors la plus utilisée dans ce cas. Chaque méthode ayant ces défauts, de nombreuses recherches sont toujours en cours sur ce thème avec notamment la prise en compte de l'anisotropie des nouveaux matériaux HTS.

\section{REFERENCES}

[1] M. N. Wilson, Superconducting Magnets, Oxford science publications, 1983.

[2] P. Tixador, Les supraconducteurs, Hermés, 1995. 
[3] C. P Bean, "Magnetization of high field superconductors", Review of modern physics, 1964, p. 31-39.

[4] Y. B. Kim, C. F Hempstead., A. R Strnad, "Critical persistent currents in hard superconductors", Phys. Rev. Lett., volume n 9 , 1962, pp. 306309.

[5] J. H. P Watson, "Magnetization of Synthetic Filamentary Superconductors, the dependence of the critical current density on temperature and magnetic field”, J. Appl. Phys., vol.39, nº pp.3406-3413.

[6] B. Douine, K. Berger, J. Leveque, D. Netter, A. Rezzoug, "Influence of $\mathrm{Jc}(\mathrm{B})$ on the full penetration current of superconducting tube" Physica C, vol. 443, pp. 23-28, 2006.

[7] B. Douine, K. Berger, J. Pienkos, J. Leveque, D. Netter, “ Analytical calculation of the instantaneous power in a current carrying superconducting tube with Jc(B)", IEEE Trans. on Appl. Supercond., vol. 18, $\mathrm{n}^{\circ} 3$, septembre 2008, pp.1717-1723.

[8] P. Vanderbemden, "Determination of critical current in bulk high temperature superconductors by magnetic flux profile measuring methods", Ph. D. Thesis, U. Lg. (1999), 193 p.

[9] S. Senoussi, "Review of the critical current densities and magnetic irreversibilities in high Tc superconductors", Journal de physique III, Vol. 2, 1992, pp. 1041-1257.

[10] B. Douine, K. Berger, J. Leveque, D. Netter, O. Arbey, N. Barthelet, "Self field effect compensation in a HTS tube", IEEE Trans. on Appl. Supercond., vol. 18, n³, septembre 2008, pp.1698-1703.

[11] B. Douine, J. Leveque, S. Mezani , "J $\mathrm{J}_{\mathrm{C}}(\mathrm{B})$ determination method with the help of the virgin magnetization curve of a superconducting cylinder", I.E.E.E. transactions on applied superconductivity, vol. 20 (2), pp.82-86, 2010 . 